\title{
Identification of Malicious Nodes \& Paths to Reduce Packet Loss in Mobile ADHOC Network with NS2 Simulator
}

\author{
P.Haritha, R.Puviarasi
}

\begin{abstract}
System security assumes a vital job in this MANET and the customary method for ensuring the systems through firewalls and encryption programming is never again powerful and adequate. So as to give extra security to the MANET, interruption location components ought to be included. In this paper, particular affirmation is utilized for distinguishing vindictive hubs in the specially appointed system. NS2 is utilized to recreate and assess the proposed plan and look at it against the $A A C K$. The acquired outcomes demonstrate that the specific affirmation conspire outflanks AACK as far as system bundle conveyance proportion and directing overhead. Portable Ad-hoc Network is an impermanent system made out of versatile hubs, associated by remote connections, without settled infrastructure. This paper proposes a novel component considered specific affirmation for taking care of issues that emerge with Adaptive Acknowledgment (AACK). Points of interest in this paper are security is expanded and can enhance the execution of the system.
\end{abstract}

Keywords - AODV, MANET, Malicious node, Security.

\section{INTRODUCTION}

Lately transportable ad-hoc systems (MANETs) have got big consideration due to their self-configuration and selfprotection talents. A mobile ad-hoc community [1] is an arrangement of far flung versatile hubs that regularly selforganize in discretionary and impermanent system topologies. People and cars would thus be able to be net labored in regions without a prior correspondence infrastructure or whilst the usage of such framework requires remote enlargement. Within the transportable advert-hoc, hubs can mainly speak with the various hubs interior their radio extents; while hubs that aren't inside the instantaneous correspondence make bigger utilize middle of the road hub to speak with one another. In these two circumstances, every one of the hubs that have element taken in the correspondence consequently frame a faraway device, on this manner this form of far flung gadget may be seen as flexible ad-hoc arrange. In no way like cord line arranges, the only of a kind attributes of transportable advert-hoc systems represent diverse non-insignificant problems to the security plan. Existing connection security techniques are regularly connected inside faraway systems to decrease safety dangers. Snooping is unapproved access to a person else's data. It's miles like listening in yet isn't always absolutely constrained to gaining access to facts

Revised Manuscript Received on July 18, 2019.

P.Haritha, Department of Electronics and Communication Engineering, Saveetha School of Engineering, Saveetha Institute of Medical and Technical Sciences, Chennai-602105.

Dr.R.Puviarasi, Department of Electronics and Communication Engineering, Saveetha School of Engineering, Saveetha Institute of Medical and Technical Sciences, Chennai-602105. amid its transmission. Snooping can incorporate easygoing popularity of an electronic mail that shows up on every other's pc display or watching what every other man or woman is composing. Increasingly more complex snooping makes use of programming initiatives to remotely display movement on a pc or system gadget. In device layer wormhole assault, a noxious hub receives bundles at one vicinity inside the machine and passages them to another area inside the machine, wherein those parcels are loathe into the network [2]. This passage between two plotting assailants is alluded to as a wormhole. It may be constructed up through wired connection between two conniving aggressors or via a solitary long-range faraway link.

Whatever remains of this paper is sorted out as pursues. Segment 2 presents the techniques used. Segment 3 portrays the introduction. Segment 4 presents related work about the paper. Segment 5 describes the methodology of the project. Segment 6 tells about the simulation process and flow chart of the how the process is going to be. Results are also discussed in segment 7.

Researchers built up a calculation to distinguish the pernicious hubs. In this paper another system got back to bunching based discovery utilizing off term strategy is utilized.

Bunching based methodology will first shape groups with a little gathering of hubs and after that additionally select bunch head utilizing standard methodology. Recreation analyze was led utilizing ns-2 and this strategy expanded parcel conveyance proportion and inertness and the system execution was great.

Also, researchers built up another strategy for noxious hub identification dependent on hub gathering procedure Malignant hubs are identified utilizing question approach called topk inquiry handling inside a gathering. Reproduction analyze is done utilizing qualnet5.2 and the outcomes got accomplish high precision in location of malevolent hub inside system.

A technique dependent on the amusement hypothesis was acquainted with recognize malevolent hubs in remote sensor systems. In this technique, it is accepted that the quantity of malignant hubs is substantially less than the quantity of typical hubs and every hub doesn't know about alternate hubs type. The motivation behind this paper was to distinguish strange conduct of malignant hubs. 


\section{IDENTIFICATION OF MALICIOUS NODES \& PATHS TO REDUCE PACKET LOSS IN MOBILE ADHOC NETWORK WITH NS2 SIMULATOR}

\section{METHODOLOGY}

Selective Acknowledgment (SACK) is a technique which revises this conduct even with numerous dropped sections. With specific affirmations, the records recipient can light up the sender quite plenty all portions which have arrived successfully, so the sender need retransmit just the sections that have in reality been misplaced.

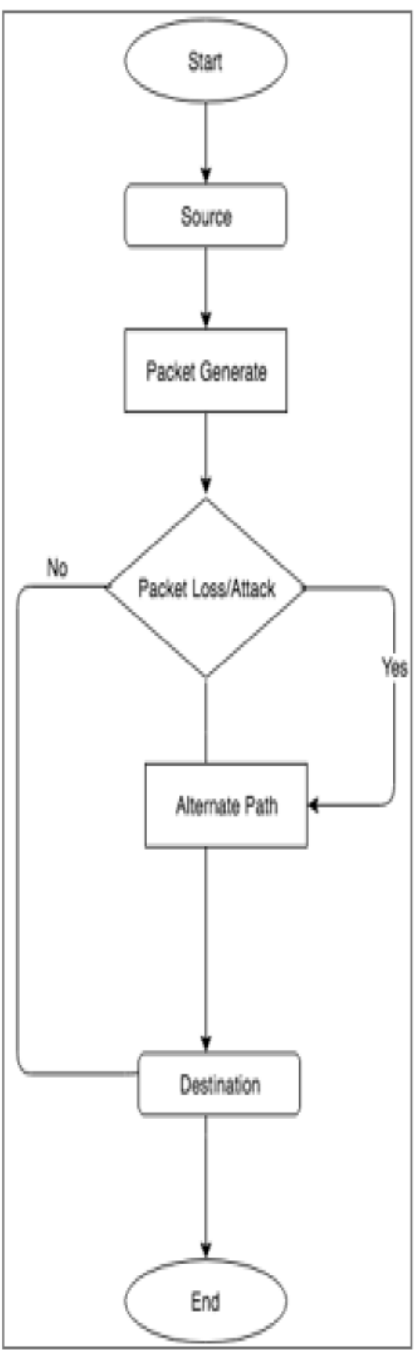

Fig1: Flowchart of proposed scheme

Bayesian induction is authentic derivation whereby proof or perceptions are utilized to refresh or to these days construe the probability that a hypothesis may be legitimate. Beta distributions, beta, are used right right here internal the Bayesian inference, because it only desires two parameters which can be constantly up to date as observations are made.

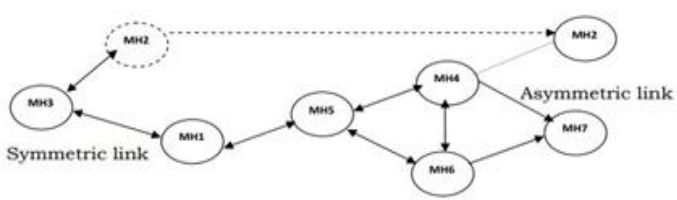

Fig 2 Mobile-hoc

\section{SIMULATION PROCESS}

The simulation is conducted using network simulator 2.35(ns 2.35) environment on a platform with Ubuntu 17.
The simulation is split into 4 clusters every which include 25 nodes.

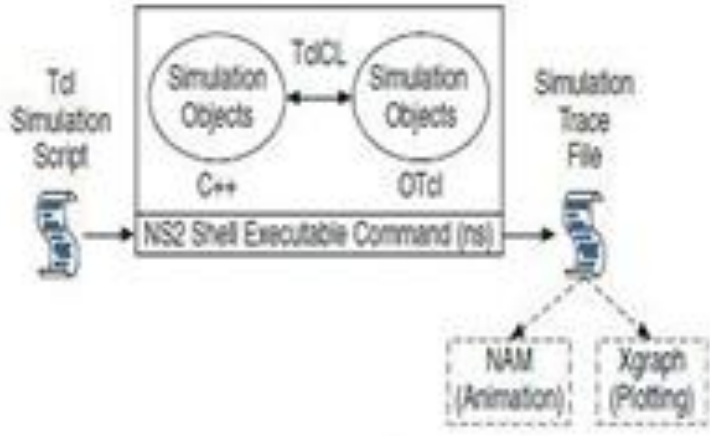

Fig 3 Architecture of NS

\section{RESULTS}

Proposed arrangement is acclimated to admit the awful bulge central the network. Packet supply arrangement and acquisition aerial is acclimated for all-embracing achievement evaluation.

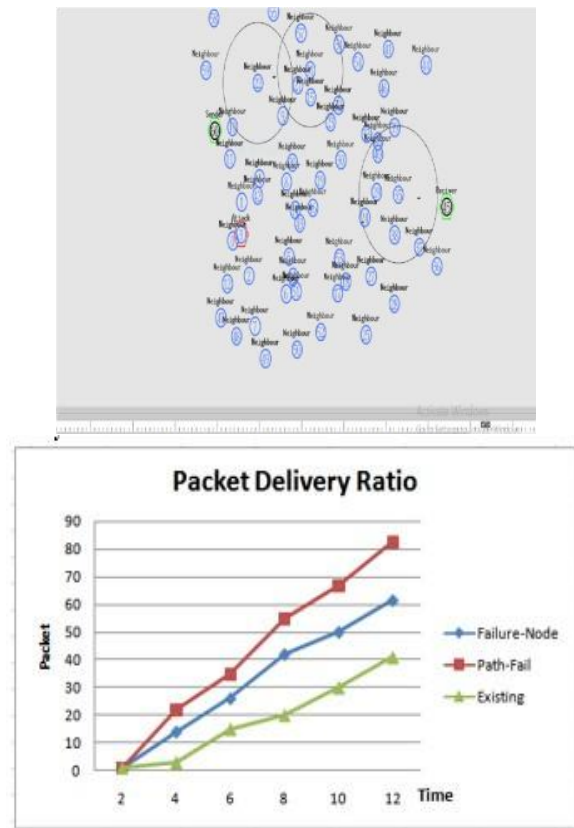

Fig4: Data Transmission and Packet Deliver Ratio

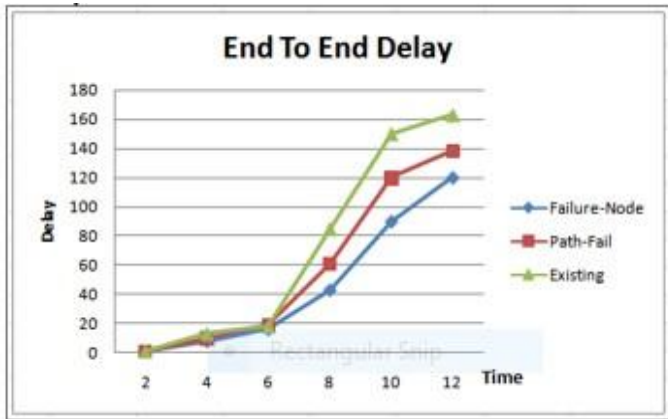

Fig5: End to End Delay 
Delay Delay is calculated using awk script which approaches the hint file and produces the end result.

\section{CONCLUSION}

The acquired outcomes demonstrate that the specific affirmation plot beats AACK as far as system bundle conveyance proportion and steering overhead. The proposed plan is utilized to distinguish and moderate the malevolent hub in the MANET. In this paper we propose a novel component considered specific affirmation for taking care of issues that emerge with Adaptive Acknowledgment (AACK). Results demonstrate that our FGA conspire beats the conventional plan with no fine grained investigation on all measurements.

\section{REFERENCES}

1. A. Abdullah Al-khatib and A. Waleedz Hammood, Mobile Malware and Defending Systems: Comparison Study, 6th vol. International Journal of Electronics and Information Engineering, 2017.

2. Lin Zhang, Xiao Song, Yunjie Wu, Theory, Methodology, Tools and Applications for Modeling and Simulation of Complex Systems, Springer, 2016. 